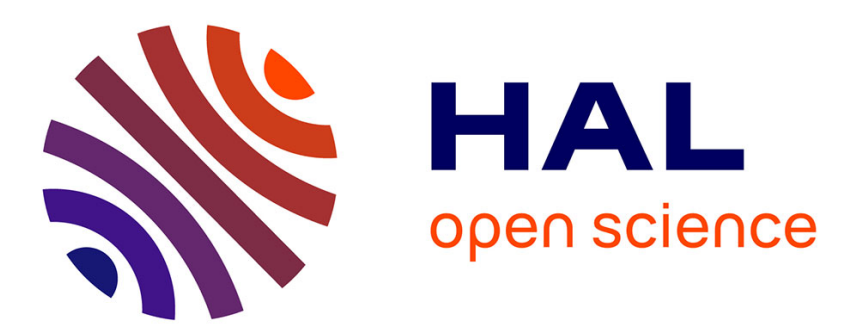

\title{
Zigzag-Shaped Superlattices on the Basis of Graphene Nanoribbons: Structure and Electronic Properties
}

\author{
V A Saroka, K G Batrakov
}

\section{To cite this version:}

V A Saroka, K G Batrakov. Zigzag-Shaped Superlattices on the Basis of Graphene Nanoribbons: Structure and Electronic Properties. Russian Physics Journal, 2016, 59 (5), pp.633-639. 10.1007/s11182-016-0816-6 . hal-01589741

\section{HAL Id: hal-01589741 \\ https://hal.science/hal-01589741}

Submitted on 19 Sep 2017

HAL is a multi-disciplinary open access archive for the deposit and dissemination of scientific research documents, whether they are published or not. The documents may come from teaching and research institutions in France or abroad, or from public or private research centers.
L'archive ouverte pluridisciplinaire HAL, est destinée au dépôt et à la diffusion de documents scientifiques de niveau recherche, publiés ou non, émanant des établissements d'enseignement et de recherche français ou étrangers, des laboratoires publics ou privés. 
${ }^{1}$ University of Exeter, Exeter, United Kingdom, e-mail: v.saroka@exeter.ac.uk; ${ }^{2}$ Research Institute for Nuclear Problems, Belarusian State University, Minsk, Belarus, e-mail: kgbatrakov@gmail.com. Translated from Izvestiya Vysshikh Uchebnykh Zavedenii, Fizika, No. 5, pp. 27-32, May 2016. Original article submitted on December $29,2015$.

\title{
ZIGZAG-SHAPED SUPERLATTICES ON THE BASIS OF GRAPHENE NANORIBBONS: STRUCTURE AND ELECTRONIC PROPERTIES
}

\author{
V.A. Saroka, ${ }^{1,2}$ K.G. Batrakov ${ }^{2}$
}

UDC 538.911, 538.915

The paper focuses on superlattices consisting of two coplanar fragments of one-layer graphene nanoribbons that have different width and are connected at an angle. Classification of such superlattices was carried out; their electronic properties were studied using the tight-binding method. It was demonstrated that in superlattices consisting of two fragments of graphene nanoribbons with armchair edges connected at an angle of $60^{\circ}$, the band gap can be regulated by the number of dimeric carbon atom chains of one of the fragments. In that case one can observe a periodic dependence of the band gap on the number of chains with a characteristic period equal to three dimeric chains. The number of dimeric chains of the second superlattice fragment regulates the average band gap value near which the periodic oscillations occur, as well as the amplitude of those oscillations. Therefore, one can accomplish a sufficiently precise band gap tuning for such structures. Such tuning can find its wide application in the booming carbon nanoelectronics industry when creating generators, amplifiers and sensors in the nanochains.

Key words: graphene nanoribbons, superlattices, electronic properties, band gap engineering.

\section{INTRODUCTION}

Over the past decade, the international research community has paid a lot of attention to graphene and its nanostructured derivatives, such as graphene nanoribbons [1-3], regarding them as the foundation for future carbon electronics [4], that is expected to replace today's silicon electronics. Electronic applications of graphene are likely to be associated with its use as a flexible substrate in VLSI schemes (VLSI stands for Very Large Scale Integration), on which the optoelectronic chains will be created by methods of nanolithography $[5,6]$. Therefore, the basic components of carbon electronics will consist of quasi-one-dimensional graphene structures that graphene nanoribbons essentially are. Today, researchers are working on the theoretical and experimental studies of their application as passive elements, namely interconnectors $[7,8]$, waveguides [9, 10], and dividers [11]. They also pay attention to the active elements of electronic circuits, such as amplifiers and switches [12-14]. The band gap of the structures used plays an important role in both cases: in the case of passive elements it is important for resistance matching, while in the case of active elements it is crucial for creation of heterojunctions. Band gap can be engineered by two methods: by doping individual ribbon sections $[15,16]$ and by engineering its shape [17-20]. Taking into account significant progress in the synthesis of graphene nanoribbons and one-dimensional structures with more complex shapes [21], this paper offers a theoretical study of the functional capabilities presented by band gap engineering in superlattices on the basis of graphene nanoribbons by means of engineering their structure.

\section{CRYSTALLINE STRUCTURE}

The crystalline structure of the examined zigzag-shaped superlattices on the basis of graphene nanoribbons is presented in Fig. 1. 


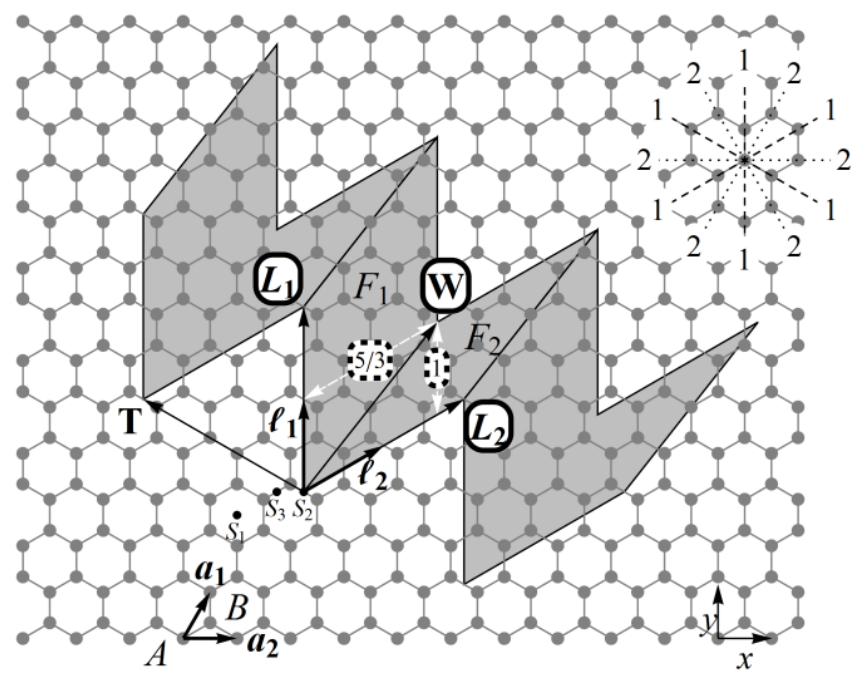

Fig. 1. Structure of the zigzag-shaped superlattice A60 $(2,2 ; 1,5 / 3)$ and the main vectors characterizing it: $A$ and $B$ are atoms from different graphene sublattices. The apex points are located on different positions $\left(S_{1}, S_{2}\right.$ and $\left.S_{3}\right)$ for

different superlattice types as indicated in Table 1.

Three vectors can describe the unit cell of such a structure: two vectors $\boldsymbol{L}_{1}$ and $\boldsymbol{L}_{2}$ that describe the shape of the superlattice edge and one vector $\boldsymbol{W}$ that characterizes its width. We shall call $\boldsymbol{L}_{1}, \boldsymbol{L}_{2}$ the shoulder vectors and $\boldsymbol{W}$ the width vector. Each of the vectors introduced can be presented by some translation vector of the two-dimensional hexagonal graphene lattice and written as follows:

$$
\boldsymbol{C}=n \boldsymbol{a}_{1}+m \boldsymbol{a}_{2}
$$

where $\boldsymbol{a}_{1}, \boldsymbol{a}_{2}$ are elementary translation vectors of the hexagonal graphene lattice, $n, m$ are whole numbers. In the general case $n$ and $m$ take independent values. Therefore, in order to describe the suggested structures, one needs to set six numbers. However, it is difficult to carry out classification in this case, so let us consider the following approach. For crystallographic directions, for which $n$ and $m$ have the greatest common divisor, vector $\boldsymbol{C}$ can be presented as follows:

$$
\boldsymbol{C}=c\left(\frac{n}{c} \boldsymbol{a}_{1}+\frac{m}{c} \boldsymbol{a}_{2}\right)=c \boldsymbol{v}
$$

where $n / c, m / c$ are whole numbers, $\boldsymbol{v}$ is some vector, let us call it "elementary vector", $c$ is the greatest common divisor for $n$ and $m$. Therefore, by means of elementary vector introduction one can fixate the crystallographic direction. This can be conveniently done for vectors $\boldsymbol{L}_{1}, \boldsymbol{L}_{2}$, in order to set the superlattice type. Two crystallographic direction are identified in the hexagonal graphene lattice; they are called zigzag and armchair. In Fig. 1, they are indicated with a dotted line 2-2 and a dashed line $1-1$ respectively. Elementary vector $\boldsymbol{v}$ has the lowest values along those directions. Let us introduce vectors $\ell_{1}, \ell_{2}$ corresponding to shoulder vectors $\boldsymbol{L}_{1}, \boldsymbol{L}_{2}$ in such a way that they would be fixated along the zigzag or the armchair direction. Such fixation could also be performed for the width vector, as it was done in $[22,23]$, but in this case it is better not to do that for the following reason. As one can see from Fig. 1, the unit cell can be presented as two fragments of ordinary ribbons $F_{1}, F_{2}$ lying in the same plane and connected at an angle, and the crystallographic direction of the vector $\boldsymbol{W}$ determines the width of fragments $F_{1}$ and $F_{2}$. That is why, in order to study the dependence of electronic properties on the width of each fragment, it is necessary to save its setting by means of two indices $w_{1}, w_{2}$. Furthermore, it is convenient to set the vector $\boldsymbol{W}$ via elementary vectors $\ell_{1}, \ell_{2}$, in 
order for indices $w_{1}, w_{2}$ to characterize the width of the corresponding fragments in length units of elementary vectors $\ell_{1}, \ell_{2}$. In the present paper let us limit ourselves to the symmetrical case, when both vectors $\ell_{1}, \ell_{2}$ are characterized by directions of the same type: either zigzag or armchair. This imposes limitations on the possible angles between them. As a result, one can distinguish four structure types: Z60, Z120, A60 and A120. The table presents the elementary vectors and a coordinate of the apex point $S_{i}(i=1,2,3)$ for each superlattice type. As it is shown in Fig. 1, the origin of coordinates coincides with one of the atoms of the $A$-sublattice.

TABLE 1 . Coordinates of elementary vectors and apex point $S_{i}$ in the basis $\boldsymbol{a}_{1}, \boldsymbol{a}_{2}$ for different superlattice types

\begin{tabular}{c|c|c|c|c}
\hline \hline $\begin{array}{c}\text { Vectors and } \\
\text { positions }\end{array}$ & Z60 & Z120 & A60 & A120 \\
\hline$\ell_{1}$ & $(1,0)$ & $(1,-1)$ & $(2,-1)$ & $(2,-1)$ \\
\hline$\ell_{2}$ & $(0,1)$ & $(0,1)$ & $(1,1)$ & $(-1,2)$ \\
\hline$S_{i}$ & $i=1 ;(-1 / 3,-1 / 3)$ & $i=1 ;(-1 / 3,-1 / 3)$ & $i=2 ;(1 / 6,-1 / 3)$ & $i=3 ;(1 / 6,1 / 6)$ \\
\hline
\end{tabular}

Within each type, a specific superlattice is determined by the set of four numbers: $\left(\ell_{1}, \ell_{2} ; w_{1}, w_{2}\right)$, where $w_{1}$ and $w_{2}$ can be fractional numbers divisible by $1 / 3$ for structures A60 and A120. Increase by 1 in one of the indices $w_{1}$, $w_{2}$ for structures Z60 and Z120 corresponds to adding one zigzag-type carbon chain to the corresponding fragment, and increase by $1 / 3$ in one of the indices for structures A60 and A120 - to adding one dimeric chain. As it is demonstrated further on, the electronic properties of superlattices can vary considerably depending on the number and type of the above-mentioned elements.

\section{ELECTRONIC PROPERTIES}

Using the tight-binding method, we have studied the dependence of the band gap of the described zigzagshaped superlattices on their geometric parameters. The calculations took into account only the $\pi$-orbits, the overlap integrals of which were set to zero. The nearest neighbors were taken into account up to the third order. Hopping integrals were selected as in [24]: $t_{1}=-3.2 \mathrm{eV}, t_{2}=0 \mathrm{eV}, t_{3}=-0.3 \mathrm{eV}$. In order to study the above-mentioned dependence, the band energy spectra of all four superlattice types were calculated for the following parameters: $\ell_{1}=5 ; 10 ; \ell_{2}=5 ; 10 ; w_{1}=2, \ldots, 31, w_{2}=5 ; 6 ; 7$.

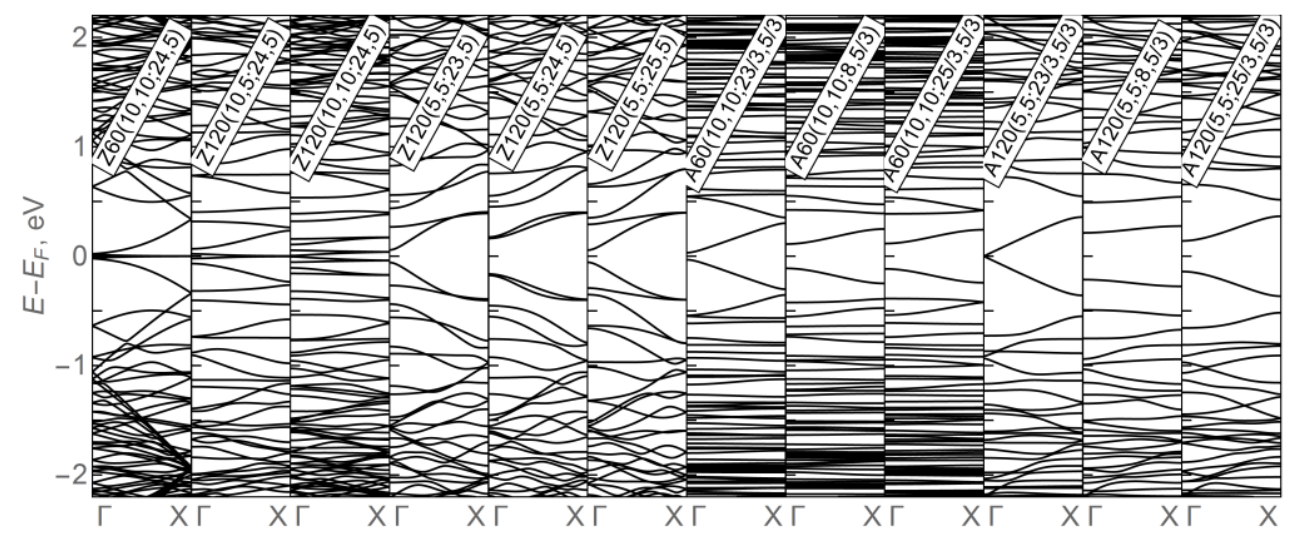

Fig. 2. Typical band spectra of zigzag-shaped graphene superlattices in the first Brillouin zone $\Gamma-X$. 
Fig. 2 presents the typical band spectra obtained by calculations. As one can see from the figure, some of the structures are metallic or almost metallic. For Z60 structures this metallicity is ensured by flat multiple-degeneracy bands that correspond to the states, the wave function of which is localized on the structure edges and on the atom closest to the apex point. Similar edge states are known for zigzag-type graphene nanoribbons [25, 26]. The same kinds of states occur for Z120 structures, but for them in many cases degeneracy is lifted and a small band gap $\sim 30 \mathrm{meV}$ is formed between the edge states. For all semiconductor structures, both wide- and narrow-gap ones, one should note the presence of the direct band gap. One should particularly note the periodic regularities for index $w_{1}$ in the band gap of some structures. In the case of small indices $w_{i}$ and large $\ell_{i}$ such superlattices are equivalent to the quantum dot chains and their spectra, as a rule, have many flat bands. However, this does not affect the periodic dependence. Let us now examine the indicated periodic dependences in greater detail, excluding Z60 metallic structures from consideration.

Fig. 3 и 4 present the results of the study of band gap dependence $\left(E_{g}\right)$ on parameters $w_{1}, w_{2}$ for superlattices Z120, A60 and A120. In all the presented cases, characteristic oscillations are observed in dependences $E_{g}\left(w_{1}\right)$. Three series were identified in each structure type for their analysis: $w_{1}=3 p ; 3 p+1 ; 3 p+2$ for Z120 and $w_{1}=p ; p+1 / 3 ; p+2 / 3$ for A60 and A120. One can see from the general picture that oscillations can be regular and irregular. In the case of regular oscillations, the series are well divided by value; the lines that can connect points within the same series are practically not intertwisted; one can identify at least a maximal or a minimal series. In the case of irregular oscillations, the lines that can connect the points within the same series are intertwisted and it is impossible to identify a minimal or a maximal series. The regular oscillations are better visualized by sequential connection of points $w_{1}=1,2,3, \ldots$ with a semitransparent line. The irregular oscillations are better visualized by connecting the points of the same series with a line.
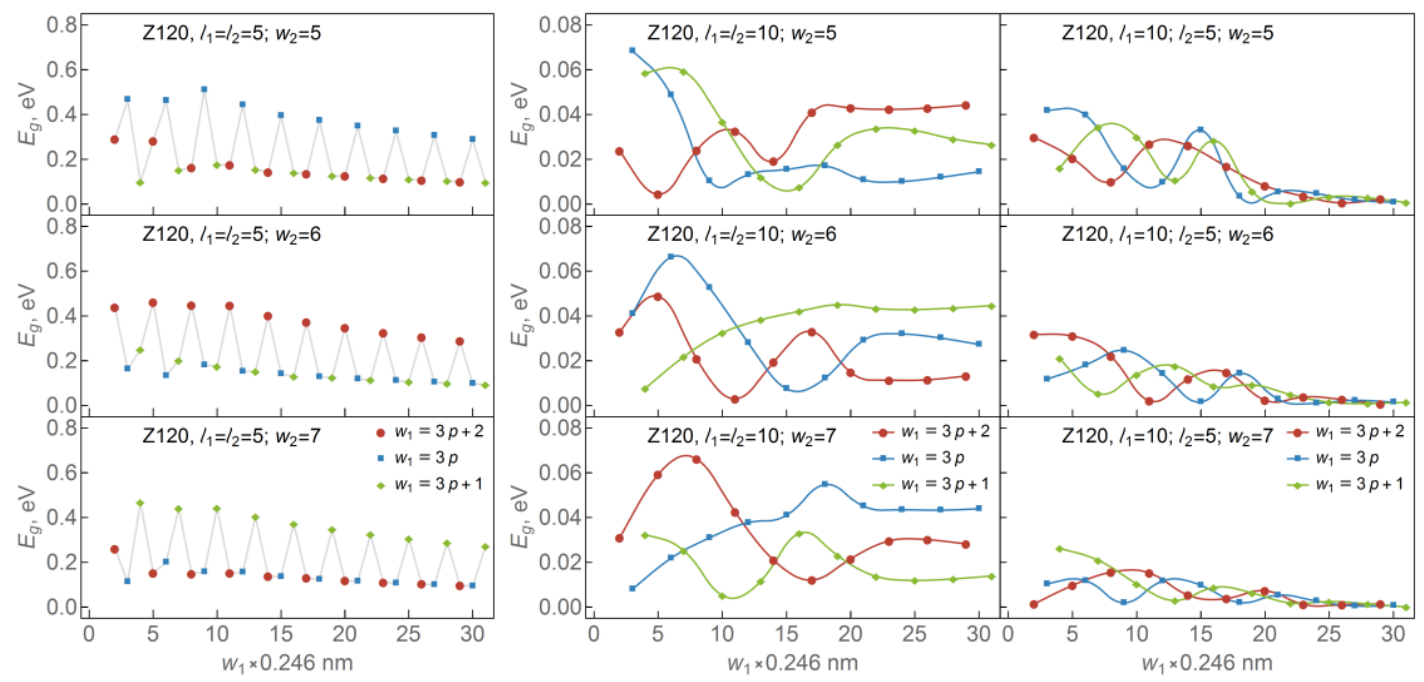

Fig. 3. Dependence of the band gap on the width indices $w_{1}, w_{2}$ for Z120 superlattices.

As one can see from Fig. 3, there are qualitative differences for small $\left(\ell_{1}=\ell_{2}=5\right)$ and large $\left(\ell_{1}=\ell_{2}=10\right)$ shoulder index values for Z120 structures. In the first case, one can observe the regular oscillations that slowly abate with the increase in $w_{1}$. One should note that it is slower than the abating $1 / w$ known for graphene nanoribbons, where $w$ is ribbon width [24, 27]. The maximal series is clearly distinguished and manifests the following dependence on index $w_{2}: w_{1}=3 p, w_{2}=3 p+2 ; w_{1}=3 p+2, w_{2}=3 p ; w_{1}=3 p+1, w_{2}=3 p+1$. In the second case, one can observe the irregular oscillations for $w_{1}<20$, that are replaced by regular ones at $w_{1}>20$. In the same way as in the 
case $\ell_{1}=\ell_{2}=5$, the amplitude of oscillations practically does not change. One should also emphasize that its absolute value is about 10 times lower and is characterized by milli-electronvolts.

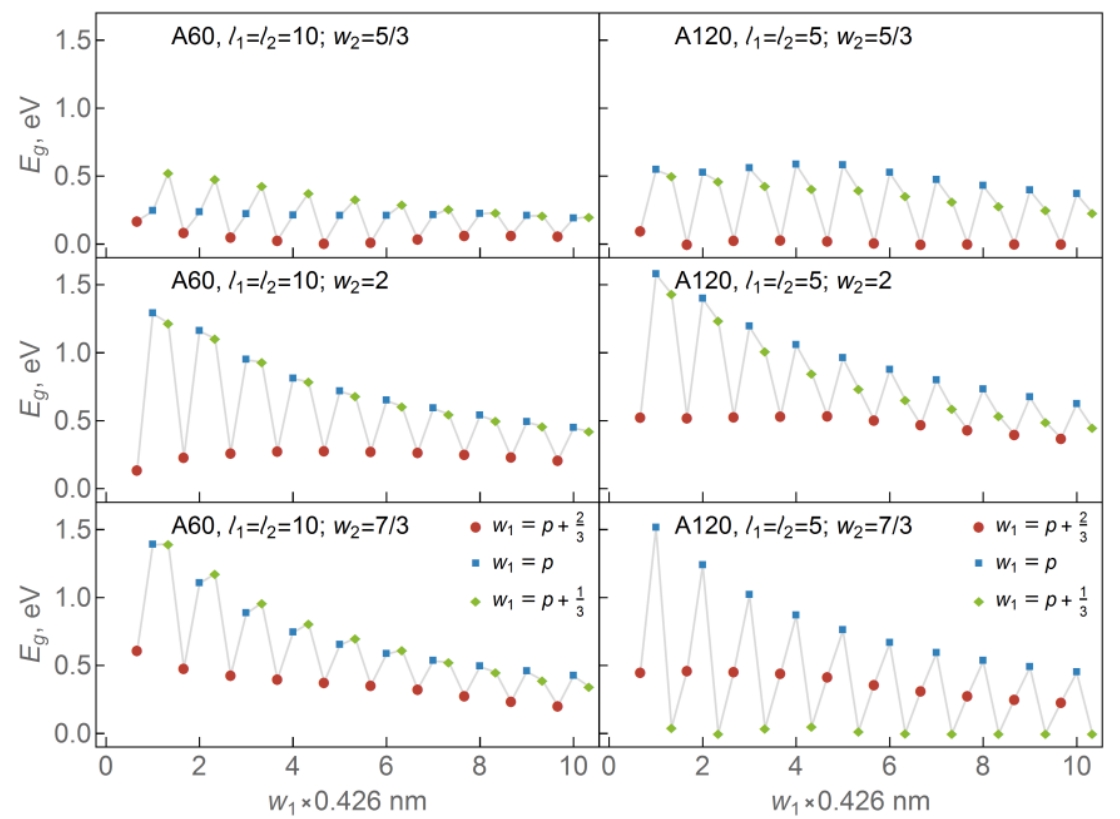

Fig. 4. Dependence of the band gap on width indices $w_{1}, w_{2}$ for superlattices A60 and A120.

As one can see from Fig. 4, superlattices A60 and A120 are characterized by regular oscillations in dependence $E_{g}\left(w_{1}\right)$. At the same time, the minimal series $w_{1}=p+2 / 3$ is clearly distinguished for A60. It is weakly dependent on $w_{1}$ and oscillates as a function of $w_{2}$. A120 structures have more complex regularities. Maximal series $w_{1}=p$ is clearly distinguished in them, while the minimal one manifests itself depending on the index $w_{2}: w_{1}=p+1 / 3$ for $w_{2}=p+1 / 3$ and $w_{1}=p+2 / 3$ in other cases.

In all the cases, the results are given for symmetrical superlattices $\left(\ell_{1}=\ell_{2}\right)$. However, one should note that the results do not change qualitatively for A60 and A120 structures and all the conclusions remain in force for the asymmetrical structures, for which $\ell_{1} \neq \ell_{2}$. In the case of Z120 structures, when $\ell_{1}<\ell_{2}$, one deals with the case close to Z60, i.e. the structures are metallic, and when $\ell_{1}>\ell_{2}$, one can observe the irregular oscillations $E_{g}\left(w_{1}\right)$ at $w_{1}<20$, that are replaced by metallic structures at $w_{1}>20$ (see Fig. 3 ).

\section{CONCLUSION}

This paper examines the new class of carbon nanostructures - zigzag-shaped superlattices on the basis of two graphene nanoribbon fragments. Classification of such superlattices was performed. The structure of the unit cells was described by means of elementary translations of the crystalline graphene lattice. The $\pi$-electron tight-binding method was used to study the dependence of the band gap on the width of ribbon fragments that make up the unit cell. It follows from the data obtained, that the simplest regularities in the band gap behavior occur for superlattices with armchair edges, where fragments connect at an angle of $60^{\circ}$. In particular, one observes the characteristic periodic dependence of the band gap on the width of one of the superlattice fragments with a period equal to three dimeric chains of carbon atoms. It, in turn, manifests the same dependence on the second fragment width. This simple combination of properties 
of ordinary graphene nanoribbons with armchair edges allows greater flexibility in regulating the band gap. After technology of synthesis of such superlattices reaches a certain level, this phenomenon should find its application when creating integrated circuits on the basis of carbon nanostructures. Let us note that optical applications in the infrared frequency range can also be possible, provided that dipole transitions between the valence and conduction bands are allowed by the corresponding optical selection rules, but this issue should become the subject of future research.

This research was supported by projects FP7 ITN NOTEDEV(FP7-607521), CACOMEL(FP7-247007), FAEMCAR (FP7-318617) and CANTOR (FP7-612285); EU H2020 RISE project CoExAN (H2020-644076); European Graphene Flagship project (604391), as well as by the Belarusian Ministry of Education (grant 20140773), Belarusian State University (grant 11, 2014), and the international grant AFOSR "Nanosized Cherenkov-Type Terahertz Light Emitter Based on Double-Walled Carbon Nanotubes and Bi-graphene Nanoribbons".

\section{REFERENCES}

1. A.K. Geim A. K. and K.S. Novoselov, Nat. Mater, 6, 183 (2007).

2. A.H. Castro Neto, N.M.R. Peres, F. Guinea, et al., Rev. Mod. Phys, 81, 109 (2009).

3. L.A. Chernozatonskii, P.B. Sorokin, A.A. Artukh, Russian Chemical Reviews, 83, Issue 3, 251-279 (2014).

4. J. Liang, Y. Chen, Y. Xu, et al., ACS Appl. Mater. Interfaces, 2, 3310 (2010).

5. $\quad$ L. Tapasztó, G. Dobrik, P. Lambin, and L.P. Biró, Nat. Nanotechnol., 3, 397 (2008).

6. Z. Wei, D. Wang, S. Kim, et al., Science, 328, 1373 (2010).

7. A. Maffucci and G. Miano, IEEE Trans. Nanotechnol., 12, 817 (2013).

8. A. Maffucci and G. Miano, Appl. Sci., 4, 305 (2014).

9. J. Christensen, A. Manjavacas, S. Thongrattanasiri, et al., ACS Nano, 6, 431 (2012).

10. R.R. Hartmann, N.J. Robinson, and M.E, Portnoi, Phys. Rev. B., 81, 245431 (2010).

11. X. Zhu, W. Yan, N.A. Mortensen, and S. Xiao, Opt. Express, 21, 3486 (2013).

12. P.B. Bennett, Z. Pedramrazi, A. Madani, et al., Appl. Phys. Lett., 103, 253114 (2013).

13. W.S. Hwang, P. Zhao, K. Tahy, et al., APL Mater., 3, 011101 (2015).

14. J.G. Son, M. Son, K.-J. Moon, et al., Adv. Mater., 25, 4723 (2013).

15. S. Blankenburg, J. Cai, P. Ruffieux, et al., ACS Nano, 6, 2020 (2012).

16. J. Cai, C.A. Pignedoli, L. Talirz, et al., Nat. Nanotechnol., 9, 896 (2014).

17. H. Sevincli, M. Topsakal, and S. Ciraci, Phys. Rev. B., 78, 245402 (2008).

18. M. Topsakal, H. Sevincli, and S. Ciraci, Appl. Phys. Lett., 92, 173118 (2008).

19. X. Wu and X.C. Zeng, Nano Res., 1, 40 (2008).

20. E. Costa Girão, L. Liang, E. Cruz-Silva, et al., Phys. Rev. Lett., 107, 135501 (2011).

21. J. Cai, P. Ruffieux, R. Jaafar, et al., Nature, 466, 470 (2010).

22. V. A. Saroka, K. G. Batrakov, L. A. Chernozatonskii, Phys. Solid State, 56, 2135 (2014).

23. V.A. Saroka, K.G. Batrakov, V.A. Demin, and L.A. Chernozatonskii, J. Phys.: Condens. Matter, 27, 145305 (2015).

24. C.T. White, J. Li, D. Gunlycke, and J.W. Mintmire, Nano Lett., 7, 825 (2007).

25. M. Fujita, K. Wakabayashi, K. Nakada, and K. Kusakabe, J. Phys. Soc. Jpn., 65, 1920 (1996).

26. K. Nakada, M. Fujita, G. Dresselhaus, and M. Dresselhaus, Phys. Rev. B., 54, 17954 (1996).

27. Y.-W. Son, M.L. Cohen, and S.G. Louie, Phys. Rev. Lett., 97, 216803 (2006). 